\title{
Praticando Qualidade de Software: Ensinando e Aprendendo seus Valores através de Ambiente Real
}

\author{
Nadja N. Rodrigues \\ Instituto Federal de Educação, Ciência e Tecnologia da Paraíba (IFPB) - Campus João \\ Pessoa - PB - Brasil \\ nadja.rodrigues@ifpb.edu.br
}

\begin{abstract}
Understanding the importance of education in software quality for integration between academy and industry, this paper reports the use of a proposal for teaching and learning aspects related to software quality. The practical side of the study was based on the instantiation of real projects of a Junior Enterprise (JE), implemented through a specific environment in the classroom. The environment allowed the students to understand the relation between quality and software processes, through the roles and activities, templates, procedures and tools used in projects development. Other contributions were the increase of JE's projects quality and the value added to students, through the use of best industry practices.
\end{abstract}

Resumo. Entendendo a importância da educação em qualidade de software para a integração entre academia e indústria, este artigo relata o uso de uma proposta para ensino e aprendizagem de aspectos relacionados à qualidade de software. O lado prático do estudo baseou-se na instanciação de projetos reais de uma Empresa Júnior (EJ), executados através de ambiente específico, em sala de aula. O ambiente permitiu que o aluno entendesse a relação entre qualidade e processos de software, através dos papéis e atividades, templates, procedimentos e ferramentas utilizados no desenvolvimento de projetos. Outras contribuições foram o aumento na qualidade dos projetos da EJ e o valor agregado aos alunos, através do uso das boas práticas da indústria.

\section{Introdução}

A indústria de software convive com a crescente busca por produtos que superem as expectativas dos seus stakeholders. Para Pressman (2006), à medida que a importância do software e a exigência por qualidade crescem, a Engenharia de Software (ES) desenvolve abordagens para melhorar o desenvolvimento e produzir software com mais qualidade. Para Sommerville (2007), a ES é uma área da engenharia voltada à produção de software, desde os estágios iniciais do sistema até a sua manutenção.

Em ES, um dos principais mecanismos para obter software de qualidade é usar um Processo de Desenvolvimento de Software (PDS). Para Carvalho et al (2011) o PDS é um conjunto de políticas, estruturas organizacionais, tecnologias, procedimentos e artefatos necessários para conceber, desenvolver, implantar e manter um produto de software. Os PDS podem ser prescritivos (mais detalhados, em suas definições e orientações, e requerem mais esforços técnicos) ou não prescritivos ou ágeis (mais simples, iterativos, com esforços técnicos simplificados). Cada modelo tem 
características definidas de acordo com cenários indicados para sua aplicação. Torna-se vital adotar um PDS que garanta a qualidade do produto gerado. Para Carvalho et al. (2011) a qualidade do PDS impacta diretamente na qualidade do produto. Segundo Sommerville (2007), a experiência tem mostrado que a qualidade do processo tem uma influência significativa na qualidade do software. O gerenciamento da qualidade do processo pode conduzir a poucos defeitos no software entregue.

Em meio à diversidade de PDS propostos pela indústria, ainda percebe-se uma dificuldade de utilizá-los em sala de aula, para ensino e aprendizagem de ES aplicada e qualidade, em virtude das diferenças entre os ambientes da academia e da indústria. Por outro lado, a academia tem, como uma de suas responsabilidades, a formação e preparação de profissionais com excelência técnica para favorecer a captação destes, contribuindo para o desenvolvimento das diversas áreas de pesquisa e setores econômicos, e consequentemente, do país. Segundo Calazans (2009), as disciplinas de Engenharia e Qualidade de Software englobam conceitos relevantes para alunos de cursos de graduação. Para a autora, visualizar e entender conceitos de ES e qualidade e a forma de utilizá-los é prioritário nos dias atuais em que a maior parte das organizações implementa produtos de software com a utilização de modelos de qualidade.

A academia deve estar próxima da indústria, em conhecimento teórico e prático, de forma a agregar valor aos seus futuros profissionais. Uma alternativa tem sido fazer adaptações aos modelos propostos pela indústria, contemplando as boas práticas da ES, mas respeitando particularidades de cada ambiente acadêmico e assim viabilizando a execução de seus projetos. Conforme Campos et al. (2011), a emergente preocupação com a educação em ES reflete a demanda por profissionais qualificados. Considerando a importância da educação em Qualidade de Software (QS) na formação de profissionais, e buscando aproximar academia e indústria, apresenta-se uma proposta para ensino de QS. A proposta se baseia em um PDS definido para uma EJ que produz softwares, e no uso de um ambiente que apoie a estratégia de ensino de ES e qualidade na prática, através do uso dos diversos recursos de um PDS, como papéis, atividades, procedimentos, artefatos e ferramentas, e contempla o desenvolvimento de projetos reais da EJ, inclusive em sala, sendo instanciada em um curso superior do IFPB.

Este artigo está estruturado da seguinte forma: as Seções 2 e 3 apresentam trabalhos e conceitos relacionados; a Seção 4, aspectos da metodologia proposta para ensino e aprendizagem de QS; a Seção 5, características do PDS utilizado e o uso desta proposta em meio acadêmico; a Seção 6, as considerações finais.

\section{Trabalhos Relacionados}

Segundo Silva et al. (2011), as Diretrizes Curriculares de Cursos de Computação do MEC reforçam a importância da assimilação dos conceitos relacionados à ES, bem como recomendam que estes conhecimentos sejam aplicados através da prática em laboratórios e estágios. Os autores relatam que pesquisas têm confirmado que os profissionais da área aprendem tópicos de ES depois da graduação, ou seja, as competências de ES não estão sendo adequadamente abordadas nos cursos, apontando a existência de contradições dos currículos em relação às necessidades da indústria. Segundo os autores, uma das causas dessa deficiência pode estar relacionada à carência de práticas educacionais alternativas à tradicional. 
Calazans (2009) explica que no ensino da ES, a assimilação de conceitos e a execução de práticas como elicitação de requisitos, documentação, PDS, entre outros, são essenciais, agregando conhecimento que será evoluído em outras disciplinas do curso. A autora explica que o estudo da QS tende a ser uma atividade monótona, e que esse conteúdo necessita ser ministrado com criatividade e inovação para despertar o interesse do aluno, uma vez que os conteúdos eminentemente teóricos desestimulam alunos a aprendê-los, deixando lacunas prejudiciais no aprendizado.

Uma vez que já foi percebida a importância do ensino prático da ES, pela academia, podem ser encontradas várias iniciativas de definição e aplicação de PDS específicos para cada contexto. Entretanto, é difícil encontrar trabalhos relacionados especificamente ao ensino da QS. Em trabalhos relacionados ao ensino da ES aplicada podem ser reconhecidos aspectos ligados à QS. Referente ao uso de PDS na academia, o XP1 [XP1 2007] é um PDS para ambiente acadêmico, baseado nas práticas de XP [XP 2012] e propõe a execução das seguintes tarefas: Análise; Planejamento; Projeto; Testes; Documentação; Integração; Gerência; Codificação e Testes Unitários. O YP [YP 2012] é baseado em modelos como XP e RUP [RUP 2012], focando aspectos dos processos ágeis. Para Garcia et al. (2004), o YP auxilia o desenvolvimento de aplicações em ambiente acadêmico e a aprendizagem dos conceitos de ES na graduação, tendo, como etapas: Definição de papéis; Conversa com o cliente; Inicialização; Planejamento; Testes; Implementação; Reuniões de acompanhamento. Conforme Paiva et al. (2004), o Praxis é destinado a projetos didáticos em disciplinas de ES, tendo como atividades: Requisitos, Análise, Desenho, Implementação e Testes. O uso de PDS específicos e adaptados aos cenários acadêmicos está se tornando uma boa prática em cursos de Computação. A expectativa em torno desta estratégia é ensinar conceitos de ES e QS de forma prática. Essas iniciativas viabilizam e motivam o desenvolvimento de projetos de qualidade, em ambientes acadêmicos, e aproximam alunos e indústria.

Referente às novas metodologias e instrumentos para ensino-aprendizagem, como uso de ambientes específicos e tecnologias, conforme Rangel et al. (2011), notamse iniciativas na produção de softwares e ambientes de apoio à aprendizagem, que são utilizados por professores, dando suporte a novas práticas pedagógicas. Para o autor à medida que professores e estudantes se apropriam desses ambientes, novas demandas vão surgindo, os requisitos se sofisticam e realimentam a pesquisa, e a ideia é que esses ambientes estejam em maior sintonia com as necessidades do conteúdo que está sendo ensinado e do seu perfil de usuário. $\mathrm{O}$ autor explica que a ausência de ambientes computacionais adequados a diferentes propostas de trabalho representa um problema para educadores. Ribeiro et al. (2009) acredita que o processo de ensino - aprendizagem deve ser concebido de forma a permitir uma perfeita integração entre as ferramentas e as ações de aprendizagem. Para este autor, o avanço da tecnologia tem estimulado o desenvolvimento das mais diversas e inovadoras formas de difundir conhecimento. Para Veras et al. (2009) avanços tecnológicos na educação tem recebido a atenção de vários pesquisadores durante os últimos anos, e a Internet tem contribuído para melhorar os ambientes educacionais trazendo mais dinâmica e interatividade, provendo ferramentas que permitem a professores e estudantes explorar a Web de forma a compartilhar conhecimento. Para Campos et al. (2011) as diversas áreas do conhecimento tem requerido o uso de ferramentas para auxílio no processo de ensino e aprendizagem, e assim, novos métodos são desenvolvidos e novos instrumentos são inseridos em sala. 


\section{Conceitos Relacionados}

\subsection{Qualidade de Software}

Sommerville (2007) explica que o gerenciamento da qualidade formalizada é particularmente importante para equipes que estão desenvolvendo sistemas grandes e complexos, mas que uma abordagem mais informal pode ser adotada para sistemas menores. Neste caso, o processo pode ser menos burocrático, pois a equipe pode se comunicar informalmente, sendo a questão principal estabelecer uma cultura de qualidade e assegurar que todos os membros da equipe tenham uma abordagem positiva para qualidade de software.

O gerenciamento de qualidade de software para sistemas de grande porte pode ser estruturado através das seguintes atividades, segundo Sommerville (2007): garantia de qualidade (estabelecimento de um framework de procedimentos organizacionais e padrões que conduzem a um software de alta qualidade); planejamento de qualidade (seleção de procedimentos e padrões apropriados deste framework, adaptados para um projeto de software específico); controle de qualidade (definição e aprovação de processos que assegurem que a equipe de desenvolvimento de software tenha seguido os procedimentos e os padrões de qualidade de projeto).

Segundo Santos e Pretz (1990 apud IEEE, 2010), a garantia da qualidade é o conjunto de atividades planejadas para avaliar os processos pelos quais os produtos são desenvolvidos ou fabricados; o controle da qualidade, por sua vez, é o conjunto de atividades planejadas para avaliar a qualidade dos produtos desenvolvidos ou fabricados. Conclui-se então que a garantia da qualidade está focada na qualidade do processo, enquanto o controle da qualidade está relacionado à qualidade do produto.

\subsection{Verificação e Validação de Software}

A verificação e validação $(\mathrm{V} \& \mathrm{~V})$ tem como objetivo mostrar que um software está em conformidade com sua especificação e atende às expectativas do cliente.

Segundo Sommerville (2007), a verificação envolve processos tais como inspeções e revisões, a cada estágio do processo de software, desde a definição de requisitos do usuário até o desenvolvimento do programa. Sommerville (2007) explica que a validação, por sua vez, tem seus maiores esforços concentrados nos testes, quando a versão operacional é testada. Sommerville (2007) cita como estágios do processo de teste: teste de componente (ou unidade), teste de sistema e teste de aceitação. O teste de componente testa os componentes individuais do sistema; o teste de sistema, a integração dos componentes para compor o sistema; o teste de aceitação, por sua vez, usa dados do cliente para teste dos requisitos do sistema. Outras classificações de testes podem ser encontradas, dependendo do tipo de teste que se deseja realizar.

\section{Metodologia}

Este trabalho se propõe a apresentar os principais elementos que relacionam ES e QS, no contexto de uma metodologia desenvolvida para ensinar aos alunos algumas boas práticas para a construção de softwares de qualidade. A metodologia foi desenvolvida com o objetivo de ensinar tanto conceitos gerais de ES, quanto conceitos mais específicos, como qualidade, de forma aplicada, e vem sendo utilizada no Curso 
Superior de Tecnologia em Análise e Desenvolvimento de Sistemas (CSTADS) do IFPB, Campus Cajazeiras. A base da metodologia consiste em fundir os conceitos teóricos tradicionais de disciplinas da área de ES com a prática na EJ formada pelos alunos deste curso, onde os principais produtos são sistemas de software.

Para que esse estudo fosse desenvolvido e a metodologia, aplicada, foram executados vários passos: foi idealizado e criado um PDS que se adequasse à realidade da EJ, o que potencializaria o desenvolvimento técnico de projetos comerciais de qualidade, por parte da EJ e ainda tornaria possível a execução dos projetos que seriam trabalhados em sala de aula; foi criado um ambiente com os elementos deste PDS, de forma que ele pudesse ser acessado tanto para a geração dos projetos da EJ quanto para as aulas; com relação aos materiais para aula, foram utilizados projetos reais da EJ, ou fictícios, sendo as aulas dadas como oficina de projetos. Explicações sobre o estudo e a metodologia serão tratadas de forma mais detalhada na próxima seção.

\section{Praticando Qualidade de Software}

Esta seção apresenta alguns detalhes sobre a metodologia proposta para ensino de QS.

\subsection{Processo de Desenvolvimento e Ambiente de Apoio à Prática em Qualidade}

Conforme citado na Seção 4 (Metodologia), a proposta elaborada baseou-se em um PDS para a execução dos projetos em sala. Foi utilizado o Simple Way Process (SWP), PDS da EJ do CSTADS. O SWP começou a ser esboçado em 2010, tendo sua primeira versão concluída em 2011. Desde as suas primeiras sugestões de elementos, este PDS vem sendo utilizado tanto na EJ como nas disciplinas de ES aplicada (que trabalha os principais conceitos de QS), e Estágio Supervisionado, no CSTADS.

Para Rodrigues e Estrela (2012a), as empresas de desenvolvimento de software têm necessidades próprias, de acordo com suas características de trabalho, tipos de projetos desenvolvidos, clientes, dentre outras. As autoras explicam que para a EJ em estudo, o desenvolvimento deve respeitar algumas particularidades da empresa, inerentes a alunos de graduação: alta rotatividade dos integrantes; a equipe não tem o mesmo horário livre; os integrantes não dispõem de 8 horas diárias de trabalho. Para Rodrigues e Estrela (2012a), o SWP inspirou-se nas boas práticas da ES e tem como base elementos do PMBoK [2008], Scrum [2012], RUP, XP, YP e XP1. Segundo as autoras, para a execução dos projetos através do SWP, foi criado um ambiente na Web, para usuários autorizados, onde são apresentados os seus elementos. Nesta seção, serão apresentados alguns aspectos deste ambiente, sendo detalhes ligados especificamente ao ensino de QS, apresentados na Seção 5.2 (Relato de Experiência).

No ambiente do SWP, a página "Quem...”, apresenta os seus papéis (Figura 1). Segundo Rodrigues e Estrela (2012b), o SWP sugere os seguintes papeis: gerente de projetos, líder de grupo, analista, desenvolvedor e testador, além do cliente.

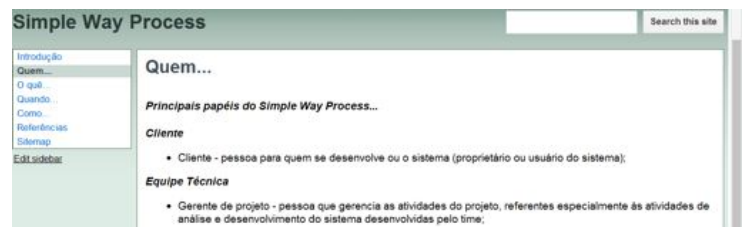

Figura 1. Ambiente do SWP - Trecho da Página "Quem..." 
Segundo Rodrigues e Estrela (2012b), para suas atividades, o SWP sugere tanto abordagens baseadas em processos prescritivos como ágeis, de forma a acomodar as práticas propostas pelos modelos às necessidades da EJ. Para exemplificar, as autoras citam o fato de a EJ ter que manter registros dos projetos bem documentados em virtude da alta rotatividade da equipe, já que o aluno concluinte deixa esta empresa. Por outro lado, a necessidade de entregas rápidas, por exemplo, é incompatível com um processo pesado. Para as autoras, o SWP avalia esses problemas e tenta equilibrar os aspectos relacionados a eles, buscando alternativas intermediárias para as suas atividades. A página "O que..." mostra as disciplinas e links para as atividades do SWP (Figura 2).

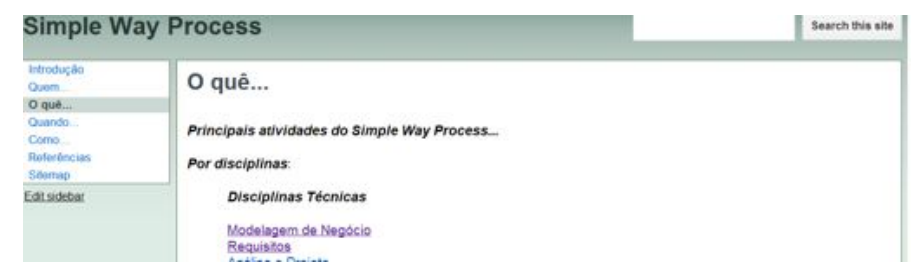

Figura 2. Ambiente do SWP - Trecho da Página "O quê..."

Segundo Rodrigues e Estrela (2012b), o SWP sugere a sua execução de forma iterativa e incremental no tempo, contemplando atividades tanto técnicas quanto gerenciais. A página "Quando...” apresenta o SWP no tempo (Figura 3).

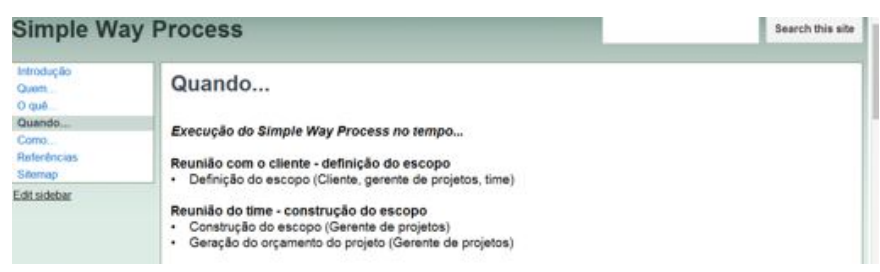

Figura 3 - Ambiente do SWP - Trecho da Página "Quando..."

Segundo Rodrigues e Estrela (2012b), o SWP define elementos para apoiar a sua sistematização. A página "Como..." (Figura 4) apresenta links para as páginas que tratam os "Procedimentos", "Templates" ou "Ferramentas": a página "Procedimentos" apresenta orientações em geral ou links para a página "Templates" (caso a orientação seja a geração de um artefato), ou para a página "Ferramentas" (caso seja o uso de alguma ferramenta); a página "Templates" exibe os templates de artefatos sugeridos; a página "Ferramentas" exibe as ferramentas sugeridas pelo SWP (todas livres).

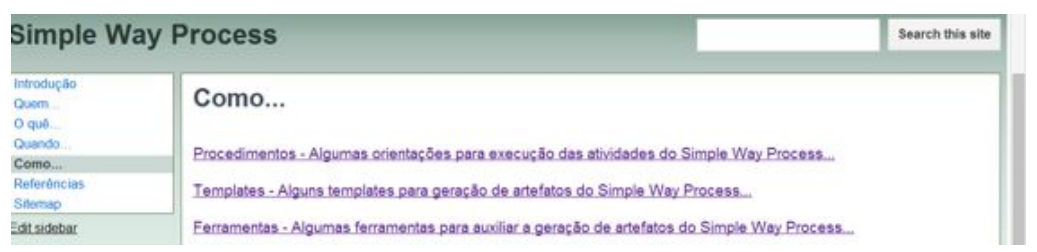

Figura 4 - Ambiente do SWP - Trecho da Página "Como..."

\subsection{Relato de Experiência}

Antes da EJ e do SWP, as aulas de ES e QS eram baseadas em modelos como RUP e XP. O RUP guiava as aulas em forma de oficina de projetos, uma vez que várias práticas do XP não poderiam ser utilizadas, pela dificuldade de os alunos trabalharem em duplas nos projetos, fora de sala de aula. Devido ao RUP possuir um grande número 
de elementos, havia uma dificuldade natural em apresentar este modelo em sala de aula, e em desenvolver todos os seus elementos, uma prática considerada inviável, para uma disciplina semestral. Alguns desses elementos eram escolhidos para ser executados nos projetos. A disciplina tinha uma alta carga teórica. Os alunos entendiam as atividades a executar, e utilizavam modelos dos artefatos ou escolhiam as ferramentas para gerá-los.

Um dos aspectos que motivou a escolha dos elementos do SWP foi a ideia de que modelos prescritivos, em geral, são considerados "pesados", enquanto modelos ágeis, "muito leves" - e neste caso requerem conhecimento apurado da equipe, o que não se observa em alunos de graduação. O SWP tenta balancear as práticas prescritivas e ágeis, para ensino de ES e aplicação de conceitos de PDS e qualidade. Antes do SWP, o nível de padronização e o grau de automação eram baixos, dificultando o desenvolvimento dos projetos. Esses projetos eram fictícios e desenvolvidos em grupos.

Conforme explicado, antes do SWP, as aulas de ES e QS eram baseadas em PDS da indústria, adaptados com dificuldade, em virtude da diferença entre indústria e academia. Desde 2010, o SWP vem sendo utilizado tanto na EJ como nas disciplinas de ES e estágio. Uma vez que a professora de ES aplicada e estágio é também consultora da EJ, alguns projetos nas disciplinas foram projetos da EJ. Os alunos que fizeram parte da EJ, na disciplina de ES, desenvolveram projetos da empresa e assim aplicaram os conceitos de ES e QS na prática em projetos. Se alunos dessa disciplina não integraram a EJ, através das aulas, estes puderam imergir na EJ, e vivenciar o trabalho na indústria de software. Alguns alunos se sentiram motivados a integrar a EJ, depois dessa experiência. Uma vez que em Cajazeiras não existem empresas de desenvolvimento, antes da EJ, os alunos não cursavam o estágio. Atualmente, estágios acontecem na EJ.

No início, o SWP sugeriu os templates. Em seguida, também ferramentas. A criação do ambiente favoreceu a imersão dos alunos nos conceitos de ES, QS e na própria EJ. A facilidade de uso do ambiente associada à padronização diminuiu a curva de aprendizado, o que favoreceu o ensino, além da execução das atividades da EJ.

Conforme explicado na Seção 2 (Trabalhos Relacionados), não é comum encontrar trabalhos voltados ao ensino de qualidade, ou disciplinas de QS em estruturas curriculares. Se os PDS forem analisados superficialmente, será percebido que vários deles não dão ênfase, explicitamente, às práticas de qualidade. Como consequência, muitas vezes, em sala de aula, a visão da qualidade é simplificada e só são tratados aspectos de padronização e testes. No cenário explicado neste trabalho, que resulta na integração de sala de aula e EJ, ou academia e indústria, tratando a geração de projetos reais, outras necessidades surgiram e derivaram variáveis de controle específicas. Uma vez que o trabalho em sala passou a utilizar projetos reais, com clientes reais, os alunos sentiram a importância de trabalhar diversos conceitos ligados à qualidade, como testes, inspeções e revisões, a partir de entregas e da constatação de bugs ou comportamentos inadequados para a aplicação, como tempos de respostas longos para buscas, por exemplo. Indicadores do uso do SWP nas disciplinas de ES aplicada (onde são vistos os aspectos de QS) e estágio, e da aplicação do SWP na EJ podem ser vistos na Tabela 1.

Tabela 1 - Alguns Indicadores do Uso do SWP

\begin{tabular}{|c|l|}
\hline Indicador & \multicolumn{1}{|c|}{ Coleta de Dados } \\
\hline Número de alunos na EJ do CSTADS $=10$ & Dados referentes à equipe da EJ, em Dezembro de 2012 \\
\hline
\end{tabular}




\begin{tabular}{|c|c|}
\hline $\begin{array}{l}\% \text { de integrantes da EJ que desejaram participar desta } \\
\text { após discussões sobre a EJ nas aulas de ES }=40 \%\end{array}$ & $\begin{array}{l}\text { Dados referentes à equipe da EJ, em Dezembro de } \\
\text { 2012, colhidos em entrevistas. }\end{array}$ \\
\hline $\begin{array}{l}\text { Número de alunos que utilizaram o SWP para as } \\
\text { práticas de QS nas aulas de } E S=42 \text { alunos }\end{array}$ & $\begin{array}{l}\text { Dados referentes às turmas 2010.2, 2011.1, 2011.2, } \\
2012.1\end{array}$ \\
\hline $\begin{array}{l}\text { Número de alunos que utilizaram o SWP para as } \\
\text { práticas de QS no estágio supervisionado }=8 \text { alunos }\end{array}$ & $\begin{array}{l}\text { Dados referentes aos estágios do CSTADS, colhidos } \\
\text { junto à Coordenação de Estágio }\end{array}$ \\
\hline$\%$ de alunos de ES (QS) que são membros da $\mathrm{EJ}=30 \%$ & Dados referentes à turma 2012.1. \\
\hline $\begin{array}{l}\% \text { de alunos de estágio (QS) que são membros da EJ = } \\
100 \%\end{array}$ & $\begin{array}{l}\text { Dados referentes à turma } 2011.2 \text { (2012.1 não teve } \\
\text { estágio). }\end{array}$ \\
\hline $\begin{array}{l}\text { Número de integrantes da EJ que utilizaram o SWP para } \\
\text { prática de QS na geração dos seus projetos = } 22 \text { alunos }\end{array}$ & $\begin{array}{l}\text { Dados referentes aos alunos envolvidos em projetos da } \\
\text { EJ desde a sua criação (2010). }\end{array}$ \\
\hline $\begin{array}{l}\text { Número de projetos da EJ gerados com o apoio do SWP } \\
\text { e das suas práticas de qualidade }=3 \text { projetos }\end{array}$ & $\begin{array}{l}\text { Dados referentes aos projetos desenvolvidos na EJ } \\
\text { desde a sua criação (2010). }\end{array}$ \\
\hline $\begin{array}{l}\% \text { de projetos da EJ gerados com o apoio do SWP e das } \\
\text { suas práticas de qualidade }=100 \%\end{array}$ & $\begin{array}{l}\text { Dados referentes aos projetos desenvolvidos na EJ } \\
\text { desde a sua criação (2010). }\end{array}$ \\
\hline $\begin{array}{l}\text { Número de projetos reais desenvolvidos no estágio } \\
\text { supervisionado com o apoio do SWP e das suas práticas } \\
\text { de qualidade }=2 \text { projetos }\end{array}$ & Dados referentes às turmas 2011.1 e 2011.2. \\
\hline $\begin{array}{l}\% \text { de alunos que fizeram o estágio baseado no SWP e } \\
\text { nas suas práticas de qualidade }=100 \%\end{array}$ & Dados referentes às turmas 2011.1 e 2011.2. \\
\hline
\end{tabular}

A seguir serão apresentados os principais aspectos do SWP relacionados à QS e ao ensino de qualidade, de forma integrada aos PDS, através da metodologia proposta.

O SWP definiu os seus papéis como gerente de projetos, líder de grupo, analista, desenvolvedor, testador e cliente. Optou-se por especializar o desenvolvimento em função das habilidades adquiridas pelos alunos no curso, em diferentes momentos. Essa abordagem viabilizou a entrada de alunos de todos os semestres na EJ, alocados nos papéis de acordo com a maturidade no curso. Nas aulas os alunos desenvolveram os projetos em grupo, executando o rodízio entre os papéis, de forma a conhecê-los.

O SWP se preocupou com aspectos de gerência de qualidade, embora não tenha pretendido realizá-la de forma muito burocrática. Quanto à qualidade do processo, o SWP definiu padrões de processo, através das sugestões de atividades e de quando e como deveriam ser realizadas, sendo dadas as orientações (e modelos) para sua realização. Referente às atividades, o SWP sugeriu tanto práticas baseadas em processos prescritivos como ágeis, envolvendo atividades referentes a disciplinas técnicas, Modelagem de Negócio, Requisitos, Análise e Projeto, Implementação, Testes e Implantação, e de apoio, Gerência de Projetos, Gerência de Mudanças e Ambiente. Na EJ, as atividades foram executadas de acordo com o papel do seu integrante na equipe. $\mathrm{Na}$ disciplina de ES, os alunos desenvolveram os projetos em grupo, fazendo o rodízio entre as atividades do SWP, de forma que pudessem conhecer o SWP e se familiarizar com as etapas da construção de software. Sendo o SWP guiado por requisitos, no estágio, cada aluno recebeu um grupo de requisitos de um projeto real e, para estes, teve que executar as disciplinas técnicas do SWP. A Gerência de Projetos foi executada por um estagiário, que por ter grande esforço nas atividades de gerência, recebeu um menor número de requisitos ou requisitos de menor complexidade, ao longo do estágio.

Referente à execução das atividades, o SWP definiu procedimentos, templates e ferramentas. Referente aos padrões, os elementos apresentados no site direcionaram o 
desenvolvimento, de acordo com as diretrizes do SWP. Como estratégia para qualidade do processo, ainda foi feito o seu monitoramento, através de inspeções e revisões, para assegurar que os padrões estavam sendo seguidos. Na EJ, as inspeções e revisões foram feitas por pessoas da qualidade. Em aula, os alunos trocaram os artefatos para realizar essas atividades. Com relação à qualidade do produto, o SWP sugeriu padrões de produtos, como templates para artefatos gerados pelo processo (26 templates, desde documentos burocráticos, até documentos de gerência ou técnicos). $\mathrm{O}$ aluno acessou o template e o instanciou, de acordo com a sua necessidade. Além dos templates, o SWP forneceu orientações e ferramentas para a construção de alguns artefatos.

O SWP ainda sugeriu as atividades de revisão do processo em si, quanto aos seus elementos, como atividades, procedimentos e templates, por exemplo. Essas revisões contemplaram a avaliação das estruturas atuais de elementos, ou a relação entre estes, e aconteceram quando foram propostas mudanças. Sugestões vindas do trabalho da EJ ou do uso do SWP em sala foram analisadas. Sobre as atividades de inspeção e revisão, em geral, foi verificado se os padrões do projeto definidos foram seguidos e se o software, os artefatos e os demais documentos estavam em conformidade com esses padrões. Os desvios com relação aos padrões foram registrados como não conformidades no artefato "Registro de problemas", sendo atribuídos aos membros do projeto, para que estes realizassem ajustes. Esse procedimento foi realizado da mesma forma na EJ e em sala.

O papel da equipe de garantia de qualidade (QA - Quality Assurance), na EJ, foi executado pelo setor de qualidade, não sendo assumido por integrantes do projeto, mas por alguém da empresa, em todos os projetos. Em sala, foi apresentada a importância da QA, e os alunos exerceram algumas de suas atividades nos projetos em sala.

Por reconhecer que os padrões de processo podem trazer dificuldades ao desenvolvimento do projeto, se o processo não for prático, sugeriu-se que o SWP fosse visto como um framework. Assim sendo, a cada projeto, durante o planejamento da qualidade, foram definidos os padrões de processo e de produto, e todos os elementos do SWP que deveriam ser desenvolvidos para cada projeto específico. Essa definição tanto aconteceu na EJ como em sala de aula. Nas aulas, especificamente, foram escolhidos todos os elementos do SWP que seriam gerados para cada turma, de acordo com um brainstorming sobre as características do projeto que seria desenvolvido no período.

A seguir serão feitas algumas considerações que tratam, especificamente, $\mathrm{V} \& \mathrm{~V}$. Para isto, remeteremos a algumas práticas explicadas anteriormente. Com relação às práticas de V\&V associadas à análise estática, o SWP sugeriu a revisão ou inspeção nos artefatos gerados por cada etapa, ao longo de todo o projeto. Os documentos referentes às disciplinas técnicas foram revisados após serem gerados e antes de serem usados como entrada para as próximas atividades. Nas aulas, esta revisão consistiu na troca de artefatos e código entre os alunos, para que uns revisassem os produtos dos outros.

Sobre os testes, o SWP sugeriu que fossem desenvolvidos em três estágios: testes de unidade, de sistema e de aceitação. Com relação aos padrões, no ambiente do SWP puderam ser encontradas várias informações. Na página "Procedimentos", foram encontradas orientações para as seguintes atividades: "Construção dos cenários dos testes unitários", "Construção/Execução dos testes unitários", "Construção dos roteiros de teste funcional", "Construção/Execução dos testes automatizados", "Registro de não conformidades", "Execução dos testes de aceitação", "Correção de não 
conformidades". Referentes aos padrões de produto, na página "Templates" foram encontrados modelos para os artefatos: "Cenários de Testes Unitários", "Roteiros de Testes Funcionais", "Registro de Problemas". O SWP sugeriu que testes fossem automatizados, para que seus custos fossem reduzidos ao longo do projeto. A página "Ferramentas" sugeriu framework para testes unitários (Ex.: JUNIT) e IDE (Ambiente Integrado de Desenvolvimento - Ex.: Selenium) para testes de requisitos. A ideia foi agilizar a correção de não conformidades no projeto. Nesse caso, testes de regressão foram utilizados e testes automatizados foram reexecutados de forma mais eficaz.

Alguns comentários sobre as práticas do SWP relacionadas a testes: o SWP recomendou o uso de revisão dos casos de teste, para descobrir se havia problemas nestes; o desenvolvimento e os testes de unidade foram intercalados; os testes de integração foram feitos o maior número de vezes possível; os codificadores elaboraram os testes e testaram o código de forma incremental, à medida que este foi desenvolvido; como o desenvolvimento foi incremental, cada incremento foi testado à medida que foi desenvolvido, com testes baseados nos requisitos desse incremento; de forma semelhante aos testes funcionais, o SWP sugeriu que fosse realizado o teste de aceitação, para que o cliente verificasse se o sistema entregue estava em conformidade com sua especificação. Por ser iterativo e incremental, o SWP favoreceu que os testes não fossem sacrificados em caso de cronogramas apertados, por exemplo. Para a EJ, o SWP ainda recomendou outros tipos de testes, como o de desempenho. Embora seja importante para a indústria, a sua execução é inviável para a aula, tendo este sido visto de forma teórica, neste ambiente. Sobre o uso do SWP para testes, em sala, o aluno visualizou e experimentou cada tipo de teste, a partir do projeto que integrou, além de ter entendido a relação existente entre os testes, e entre estes e as demais etapas do PDS.

Uma das lições aprendidas do SWP foi que repetir todos os testes a cada defeito corrigido é muito dispendioso para os projetos. Seguindo sugestões de boas práticas de teste, decidiu-se implementar um mecanismo de rastreabilidade que associe os casos de testes e os componentes do sistema, de forma que apenas um subconjunto dos testes precise ser executado novamente, no caso de reparação de defeitos. O modelo de artefato que representará esta rastreabilidade e as orientações referentes à sua construção estão sendo elaborados. Ambos serão usados em sala e pela EJ, para que se entenda a importância de conhecer as dependências entre testes e componentes do sistema.

Um resumo comparativo entre os macro instrumentos utilizados na metodologia utilizada para ensino de ES e QS, antes da criação de EJ e do SWP, e aqueles utilizados na metodologia baseada no SWP, pode ser vistos na Tabela 2.

\section{Tabela 2- Comparativo entre os principais instrumentos das metodologias utilizadas para ensino de ES e QS}

\begin{tabular}{|l|l|l|}
\hline \multicolumn{1}{|c|}{ Instrumento metodológico } & \multicolumn{1}{|c|}{ Metodologia antes do SWP } & \multicolumn{1}{c|}{ Metodologia baseada no SWP } \\
\hline Disciplinas envolvidas & ES (QS) & ES (QS) e estágio \\
\hline Tipo de projeto de software & Fictício & Fictício ou real (de preferência) \\
\hline Projetos reais desenvolvidos & 0 & 3 \\
\hline PDS de apoio ao projeto & RUP (alguns aspectos) & SWP \\
\hline Equipe de apoio ao projeto & Professora & Professora; consultores e alunos da EJ \\
\hline
\end{tabular}




\begin{tabular}{|l|l|l|}
\hline Papéis de apoio ao projeto & Escolhidos alguns do RUP & $\begin{array}{l}\text { Papéis sugeridos pelo SWP, inclusive } \\
\text { cliente real (no caso de projetos reais) }\end{array}$ \\
\hline Atividades de apoio ao projeto & Escolhidas algumas do RUP & Atividades sugeridas pelo SWP \\
\hline Templates disponíveis & Escolhidos alguns do RUP & $100 \%$ dos templates sugeridos pelo SWP \\
\hline Ferramentas & Escolhidas pelo aluno & $100 \%$ das ferramentas sugeridas no SWP \\
\hline $\begin{array}{l}\text { Orientações } \\
\text { desenvolvimento dos elementos } \\
\text { do projeto }\end{array}$ & Professora & $\begin{array}{l}\text { Professora; orientações do SWP (para } \\
\text { execução de procedimentos, geração de } \\
\text { artefatos e uso de ferramentas) }\end{array}$ \\
\hline $\begin{array}{l}\text { Padronização } \\
100 \% \text { dos templates e ferramentas do } \\
\text { SWP }\end{array}$ \\
\hline Ambiente de apoio ao projeto & Sugestões de alguns templates & Ambiente do SWP (site na Web) \\
\hline
\end{tabular}

De forma geral, pode-se dizer que as equipes conseguiram executar a prática em qualidade, através do SWP e da integração entre sala de aula e EJ, e não se depararam com maiores problemas, pois o processo estava exposto de forma clara e de fácil acesso, através do seu ambiente, para todos os alunos envolvidos nos projetos. Os principais problemas encontrados foram relacionados à inexperiência dos alunos na utilização de um processo ao longo de todo o desenvolvimento de software e de ferramentas específicas de apoio ao desenvolvimento, sugeridas pelo SWP, e do entendimento das práticas de qualidade. Outros problemas encontrados foram contornados com pequenas melhorias no processo. $\mathrm{O}$ fato de estes alunos terem compartilhado conhecimento com os demais integrantes da EJ, vários destes já com mais maturidade no SWP e nos projetos, ajudou a resolver ou minimizar o impacto destes problemas.

Acredita-se que a proposta apresentada neste trabalho traz objetividade e simplicidade ao ensino de QS através do uso do SWP, ressaltando a importância da prática da qualidade de forma integrada aos princípios de PDS.

\section{Considerações Finais}

Uma proposta para ensino e aprendizagem de QS, integrada a aulas de ES aplicada, foi apresentada neste trabalho, visando inserir a prática em qualidade como recurso complementar à teoria vista em aula. A proposta tem como base o uso de PDS adequados às características de cada IES, e de ambientes que os suportem, de forma que os alunos vivenciem as boas práticas da indústria de software integradas à qualidade de seus produtos, através do desenvolvimento de projetos, em sala de aula.

O estudo foi desenvolvido junto ao CSTADS. Utilizou-se o PDS da sua EJ, o SWP, para ensino e aprendizagem de QS. O SWP foi utilizado como recurso para apresentação das boas práticas em qualidade, $\mathrm{V} \& \mathrm{~V}$ e, especificamente, testes, através das disciplinas ES e estágio. Os alunos entenderam os diversos aspectos de qualidade estudados, através da visão da sua aplicação, e ainda a forma como estão integrados ao PDS, tendo a visão do todo. A ideia da proposta é que a qualidade seja vista como viável, e se torne meta para o desenvolvimento de software, desde o ambiente acadêmico, sendo levada como lição aprendida para a indústria, incentivando empresas e profissionais da área a usá-la. A estrutura e o ambiente do SWP e a metodologia aplicada nas disciplinas, através da imersão dos alunos em um ambiente real da indústria de software (a EJ), favorecem a prática dos aspectos de qualidade. O fato de serem instanciados projetos reais potencializa a necessidade de aplicação dos aspectos de qualidade. 
A experiência de ensino e aprendizagem de ES e dos aspectos de QS através do SWP tem como ponto forte o fato de favorecer o contato dos alunos com as rotinas de uma empresa de desenvolvimento, executando projetos através das boas práticas da indústria. Outros pontos positivos são a cooperação dos alunos de ES e estágio para a melhoria contínua do SWP, e a capacitação de atuais ou potenciais membros da EJ (oriundos das disciplinas). Pode-se pensar nos benefícios para a indústria, que passa a ter à sua disposição profissionais mais próximos das referências de excelência técnica, devido ao valor agregado aos alunos através de boas práticas da ES. Como trabalho futuro, espera-se executar o refinamento do SWP. Avalia-se a possibilidade de criação de uma equipe de colaboração para ampliação do uso do SWP, que deve ser estendido às demais disciplinas.

\section{Referências}

Campos, B. et al. F. (2011). "Experiência de Projeto e Desenvolvimento de Jogo para Ensino de Engenharia de Requisitos para Sistemas Ubíquos". XXII SBIE.

Calazans, A. T. S. (2009). "A andragogia e a criatividade como facilitadores na aprendizagem da Engenharia e da Qualidade de Software". XVII WEI.

Carvalho D. D., et al. (2011). "Apoio à Reutilização de Processos de Software em um Ambiente de Engenharia de Software Centrado em Processo". X SBQS.

Garcia, F. P., et al (2004). "easYProcess: Um Processo de Desenvolvimento para Uso no Ambiente Acadêmico". XII WEI, XXIV CSBC.

IEEE (1990), Standard Glossary of Software Engineering Terminology. IEEE.

Paiva, D. M. B, et al (2004). "Definindo, Implantando e Melhorando Processos de Software em Ambiente Acadêmico". VI Simp. Internacional de Melhoria de Processos de Software.

PMBok (2008). PMBoK GUIDE - Project Management Body of Knowledge.

Pressmam, R. S. (2006). Engenharia de Software. 6 ed. São Paulo: McGraw-Hill.

Rangel, V. G. et al. (2011). "VCom: Uma Abordagem para Modelagem de Ambientes Colaborativos para Apoiar a Aprendizagem". XXII SBIE.

Ribeiro, P. S., Franciscato, F. T., Mozzaquatro, P. M., Medina, R. D. (2009). "Validação de um Ambiente de Aprendizagem Móvel em Curso a Distância”. XX SBIE.

Rodrigues, N.N., Estrela, N.V.A.(2012a). "Simple Way: Ensino e Aprendizagem de Engenharia de Software Aplicada através de Ambiente e Projetos Reais". VIII SBSI.

Rodrigues, N.N., Estrela, N.V.A.(2012b). “SimpleWayProcess: Da Academia à Indústria de Software". XXXII CSBC.

RUP (2012). Rational Unified Process. http://www.wthreex.com/rup/portugues/index.htm.

Santos, L. B., Pretz E. (2010). "Framework para Especialização de Modelos de Qualidade de Produtos de Software". IX SBQS.

Scrum (2012). Scrum Alliance. http://www.scrumalliance.org/. Janeiro.

Silva, T. G. D., et al. (2011). "Abordagem de Apoio ao Ensino e Aprendizagem de Teste de Software Baseada em Jogos Sérios e Mundos Virtuais”. XXII SBIE.

Sommerville, Ian. (2007). Engenharia de Software. São Paulo: Pearson Addison-Wesley.

Veras, D. et al. (2009). "Uma Arquitetura para Integrar Ambientes Educacionais na Web com Sistemas em T-Learning”. XX SBIE.

XP (2012). eXtreme Programming. http://www.extremeprogramming.org. Janeiro.

XP1 (2007). "XP1: Um Processo de Desenvolvimento". http://dsc.ufcg.edu.br/ jacques.

YP (2012). eas YProcess. http://www.dsc.ufcg.edu.br/ yp. Janeiro. 